\title{
SYNERGISTIC EFFECT OF RESVERATROL WITH 5-FLUOROURACIL IN CHEMOTHERAPY OF INDUCED ORAL SQUAMOUS CELL CARCINOMA
}

\author{
Dina Ashraf Ibrahim El-Oraby ${ }^{1}$, Magda Mohamed Aly Hassan², Marwa Mokbel El-Shafei ${ }^{3}$, \\ Wafaa Hassanein El-Hossary ${ }^{4}$
}

DOI: $10.21608 /$ dsu.2020.30956.1038

Manuscript ID: DSU-2005-1038

\section{KEYWORDS}

Chemotherapy;

Fluorouracil; $I H C$;

Oral Squamous Cell

Carcinoma;

p53; Rat tongue; Resveratrol.

- E-mail address: dinaelorabyy@gmail.com

1. Postgraduate student of Oral Pathology, Faculty of Dentistry, Suez Canal University, Ismailia, Egypt

2. Professor of Oral Pathology, Faculty of Dentistry, Suez Canal University, Ismailia, Egypt

3. Professor of Oral Pathology, Faculty of Dentistry, Misr International University, Cairo,Egypt

4. Associate Professor of Oral Pathology, Faculty of Dentistry, Suez Canal University, Ismailia, Egypt

\section{ABSTRACT}

Introduction: Oral cancer is a common cause of death in human, its treatment involves chemotherapy. However, toxicity is an obstacle to this treatment. Studies suggested that combining chemotherapy with specific antioxidants can improve drug efficacy and reduce the chemotherapeutic drug toxicity. Aim: To evaluate the anticancer effect of resveratrol alone or combined with 5-Fu on experimentally induced tongue cancer. Materials and Methods: Forty male albino rats were divided into five groups: Group I: untreated (negative control), group II: positive-control, receiving 50 ppm 4-NQO in drinking water, group III: received i.p injection of (5-FU), group IV: oral-administration of resveratrol (RV), and group V: receiving both RV and 5-FU. Before euthanasia, blood samples were withdrawn to evaluate serum level of ALT, AST and creatinine. After euthanasia, the tongues were surgically excised, and processed for $\mathrm{H} \& \mathrm{E}$, and mutant p53 IHC. The mean of positive cells/group were statistically analyzed. Results: Group I showed normal tongue mucosa with negative immune expression of mutant p53 cells. Group II revealed malignant ulcers in a form of well/moderately differentiated SCC, and the highest mean of mutant p53 immuno-expression. Group III showed mild dysplasia of the tongue epithelium with a significant regression of the mean number of mutant p53 positive-cells. Group IV expressed moderate dysplasia, with a significantly high mean of mutant p53. Group V showed mild dysplasia with comparable mean of mutated p53 positive cells. Regarding toxicity, group II showed the maximum elevation of ALT, AST, and serum creatinine. These levels were decreased significantly in the treated groups. Conclusion: Resveratrol has a promising therapeutic effect against 4NQO- induced tongue cancer as 5-FU alone. It diminished the cytotoxic effect of 5-FU and enhanced normal histologic-appearance of tongue mucosa when used in combination.

\section{INTRODUCTION}

Head and neck cancer (HNC) is one of the most common causes of death in human. In most cases, its treatment involves chemotherapy ${ }^{(1)}$. However, the drug toxicity and/or tumor cell resistance are obstacles to the choice of chemotherapy ${ }^{(2)}$. Scientific studies proved that combining chemotherapy with specific antioxidants, at defined dosages can improve drug efficacy and/or may reduce the severity of side effects ${ }^{(3,4)}$. 
The most commonly used chemotherapeutic drug is 5-fluorouracil (5-FU) in treatment of oral, breast, stomach and pancreatic cancer, that remains the cornerstone of chemotherapy ${ }^{(5,6)}$. It has been widely used in combination with other drugs as well. Many of these effective drugs have been developed from botanical sources ${ }^{(7)}$.

Due to the side effects of 5-FU in chemotherapy, an introduction of a natural extract had been used in different in vivo studies to enhance the efficacy of chemotherapy, reduce the treatment duration, reduce the expected dose and in turn its toxicity. ${ }^{7}$ Resveratrol (RV) is one of the natural extracts that plays a synergistic effect with 5-FU, as revealed in a recent study on rat colorectal cancer ${ }^{(4)}$. It's effects include antibacterial, antifungal, antioxidant (through scavenging reactive oxygen species $(\mathrm{ROS})^{(8)}$, stimulates cell cycle arrest, that in turn stimulates apoptosis ${ }^{(9)}$. It inhibits invasion and metastasis through modulation of ecto-mesenchymal transition ${ }^{(10)}$. Furthermore, it leads to protein kinases inhibition, antiapoptotic gene expression, as well as angiogenic, metastatic gene products and inflammatory biomarkers ${ }^{(11)}$.

P53 is an intensively studied tumor suppressor gene. When mutated, it leads to loss of wild type $p 53$ activity. ${ }^{12}$ Disturbances in P53 signaling pathways are believed to be required for the development of most cancers, and there is evidence to suggest that its restoration or reactivation will lead to a therapeutic benefit ${ }^{(12-14)}$.

For studying oral cancer prevention and treatment, one of the most commonly used animal model is cancer induction in the rat tongue, by the water-soluble 4-nitroquinoline1-oxide (4NQO) ${ }^{(15)}$. Chronic administration of $4 \mathrm{NQO}$ induces rat tongue cancer at the same manner that occurs in humans, in terms of initiation, promotion, and progression ${ }^{(16)}$.
The mechanism of 4NQO- induced malignancy is through reactive oxygen species (ROS) generation, such as superoxide radicals, hydrogen peroxide and reactive nitrogen species (RNS) ${ }^{(17)}$. The damage induced by ROS and RNS are direct and indirect to macromolecules, including $\mathrm{DNA}^{(18)}$. The mentioned damages are almost the same as those caused by other carcinogens present in tobacco smoking, which is one of the most common risk factors for human OSCC ${ }^{(17,19)}$.

In the present study, the anticancer effect of resveratrol combined with 5-FU has been assessed on experimentally- induced tongue cancer in rats, which could be a promising alternative for resistant oral cancers.

\section{MATERIAL AND METHODS}

Approval of the research ethics committee (REC), Faculty of Dentistry, Suez Canal University was obtained before starting the study (129/2018).

Chemicals: 1. Carcinogen (The 4-Nitroquinoline 1-oxide (4NQO) (Sigma-Aldrich, USA), solution in a concentration of $0.005 \%, \mathbf{2}$. Resveratrol (RV) (Sigma-Aldrich, USA), 3.Fluorouracil: 5-Fluoro2,4(1H,3H)-pyrimidinedione (5-FU) (Pfizer lnc. Company, USA). 4. Dimethyl sulfoxide (DMSO) (Pfizer lnc. Company, USA).

Animals: Forty male albino rats weighing 150$170 \mathrm{gm}$ average 5-7 months of age. Rats were kept five per cage in a well-ventilated room with controlled temperature, 50-70\% humidity and 12 hours day/dark cycle. They were given water and recommended diet ad libitum.

\section{Grouping and treatment protocol:}

The animals were divided in groups as follows:

Group I: Negative control group: five animals were euthanized at day zero 
Group II: Positive control group: five animals were treated with 4-Nitroquinoline (4NQO) in drinking water in a dose of $50 \mathrm{ppm}(0.005 \%)$ for twenty weeks, then were euthanized a day after the twentieth week.

Group III: Ten animals were intraperitoneally (i.p) injected by 5-fluorouracil (5-FU) in a dose of $40 \mathrm{mg} / \mathrm{kg}$, 3 days weekly, day after day,for two months.

Group IV: Ten animals treated with resveratrol (RV) orally administrated dissolved in DMSO in a dose of $200 \mu \mathrm{g} / \mathrm{kg}$ body weight daily for two months.

Group V: Ten animals treated with both 5-fluorouracil (i.p.) in a dose of $20 \mathrm{mg} / \mathrm{kg} 3$ days a week (day after day, for 2 months) and resveratrol orally $(100 \mu \mathrm{g} / \mathrm{kg} /$ body weight daily for two months).

\section{Hematologic investigation and euthanization}

Liver and kidney toxicity were assessed through measuring ALT, AST and serum creatinine levels, following cancer induction and treatment. Blood samples were withdrawn before euthanasia as follows:

Each animal was anaesthetized in a closed glass container with cotton piece soaked with diethyl ether, Fine capillary tube was introduced into the orbital sinus through the mesial angle of the eye. Blood sample $(2 \mathrm{ml})$ was withdrawn into a blood collection tube with liquid EDTA and immediately sent to the laboratory.

Euthanasia method: Animals were euthanized by inhalation of diethyl ether (lethal dose of cotton piece soaked with diethyl ether).

\section{Histologic sections preparation}

The tongue was excised from each animal and grossly inspected to evaluate any apparent lesions. Tongues were fixed in $10 \%$ neutral formalin solution, processed, embedded in soft paraffin, sectioned serially into $5 \mu \mathrm{m}$, and stained with hematoxylin and eosin (H\&E) for histopathologic analysis.

\section{Histopathologic evaluation}

The H\&E stained sections were blindly diagnosed by two pathologists. Histologic grading of dysplasia was classified according to the WHO $(2017)^{(20)}$, while, histological grading of carcinoma followed Broder's classification ${ }^{(21)}$.

\section{P53 Immuno-histochemical staining (IHC)}

Five $\mu \mathrm{m}$ thick sections were cut and picked up on positively charged slides for p53 immunohistochemical (IHC) staining procedure. The antibody used was p53; a mouse monoclonal antibody Ab-1 (Clone PAb 240). The detection system used was UltraVision Detection System Anti-polyvalent, HRP/DAB. Both from Neo Markers Lab Vision Corporation, Westinghouse, Fremont, USA. Staining protocol was performed as instructed by the manufacture.

\section{P53 scoring}

Stained tissue sections were examined and assessed by using Image $\mathbf{J}$ Soft Imaging Analysis System. Three slides from each tongue were IHC stained, then three fields from each slide were randomly counted for the mean of positive cells of each slide. Adding the mean of all 3 slides to get the mean of positive cells/group. A standard measuring frame of counted cells was used, to count positive cells (nuclear stain), and statistically analyzed. 


\section{Statistical analysis}

Measurements were expressed as means \pm standard deviations. The statistical differences of each treated group compared to negative and positive control groups (group I and group II), were evaluated by One-Way Analysis of Variance, and descriptive tests (ANOVA). A $p$ value of $\leq 0.05$ was considered as statistically significant difference.

\section{RESULTS}

\section{Clinical findings}

Group I (Negative control): Showed no gross changes. Hair appeared normal, and the animals looked healthy and active throughout the experimental period.

Group II (Positive control : Five rats received 4NQO only for 20 weeks, showed multiple tongue ulcerated lesions with necrotic debris and indurated base. Debilitation of all animals was a noticeable remark, with extreme weight loss.

Group III: Ten animals (received 4NQO for 20 weeks followed by 5-FU for two months) suffered from multiple tongue ulcerations measuring about 2-3 $\mathrm{mm}$ on the dorsal surface, general edema and erythema of the tongue, as well as less extensive necrotic debris till week twenty. They slightly recovered by end of the experiment after treatment; in comparison to group II. Ulcerations were partially reduced in size/number during the fourth week of treatment, measuring about $1.5-2 \mathrm{~mm}$ and regressed to $0.5-1 \mathrm{~mm}$ at end of treatment. Two rats died at the fourth week of cancer induction, and three at the second and third weeks of treatment. They were compensated with other rats to get the same count (10 rats).

Overall, the rats showed better activity, diet consumption and gained some of their weight as compared to group II.
Group IV. (Ten rats receiving resveratrol only after cancer induction by $4 \mathrm{NQO}$ ) showed significant weight loss as that detected in group II during cancer induction and didn't totally recover by end of the experiment. Necrosis and ulcerations were less extensive than in group II. The ulcers were smaller with reduced edema and erythema of the tongue. The ulcers measuring about $1.5-2 \mathrm{~mm}$ after the first month of treatment and regressed to approximately $1-1.5 \mathrm{~mm}$ in diameter at end of the eighth week of treatment (end of experiment). Comparing to group II, there was a noticeable better activity and weight gain almost comparable to group III. However, four animals died by the seventh week of cancer induction and other two during the first and second weeks of treatment. They were compensated by other animals.

Group V. (Ten rats receiving $4 \mathrm{NQO}$ for cancer induction, followed by treatment with both 5FU and RV in half the doses given in groups III and IV). The animals showed the least necrotic tongue debri and ulcerations regarding size and number after the first month of treatment. The general tongue architecture was getting better by end of treatment, as the ulcers were markedly less in number and size than groups II, III and IV. However, six out of ten rats died during the tenth and fifteenth week of cancer induction and were compensated with other animals. They regained their normal activity, and diet consumption, as reflected by gaining most of their weight.

\section{Hematologic findings.}

According to the results, it was found that group II showed the maximum elevation of ALT and AST reflecting liver toxicity, as well as the creatinine level, reflecting kidney toxicity compared to group I. These levels were decreased significantly in the treated groups; where group III (5FU) showed a 
significant decrease in liver and kidney enzymes compared to group II, but high as compared to the untreated animals (group I).Animals treated with RV only (group IV) or in combination with 5-FU (group V) showed significant decrease in liver and kidney enzymes compared to group II (positive control), with almost comparable values to the negative control (group I).

\section{Histopathological (H\&E) and immune histo- chemical findings (IHC):}

Group I (negative control): The features of normal tongue mucosa were revealed as keratinized stratified squamous epithelium with rete processes, and a normal appearing filiform papillae. The underlying submucosa consists of a layer of dense collagenous fibrous tissue (lamina propria) devoid of inflammatory infiltrate and dense striated muscle fibers. (Figure 1A, B). The IHC findings, of p53 expression, showed negative mutant p53 positive cells. (Figure 1C)

Group II (4NQO only for 20 weeks): Overall H\&E histopathological findings showed focal malignant ulcers representing well/moderately differentiated squamous cell carcinoma; with invading islands to the connective tissue and muscle fibers. The rest of the tongue showed areas of epithelial hyperplasia and hyperkeratinization with severe dysplastic changes. Loss of polarity and hyperplasia of basal cell layer, cellular and nuclear pleomorphism, nuclear hyperchromatism and prominent nucleoli, altered nuclear/cytoplasmic ratio, increased normal and abnormal mitotic figures, dyskeratosis (individual or group cell keratinization) and swirling pattern of spinous layer representing the dysplastic changes. The underlying connective tissue was collagenous, showing intense chronic inflammatory cellular infiltrate and dilated blood vessels (Figure 2). Regarding (IHC), the highest mean of mutant p53 positive cells, with expression along the epithelial thickness and the invading islands in the connective tissue (Figure 3).

Group III (5-FU for 2 months after 4NQO for 20 weeks): Overall H\&E findings showed epithelial hyperplasia and hyperkeratinization with mild dysplastic changes in the lower third as, basal cell hyperplasia, drop shaped rete ridges, loss of polarity of basal cells, nuclear hyperchromatism, as well as nuclear and cellular pleomorphism. The underlying connective tissue showed chronic inflammatory cellular infiltrate and dilated blood vessels (Figures 4A,B). Immunohistochemistry revealed a significantly lower mean of mutant p53 positive cells compared to group II, and confined to the basal cell layer. (Figure 4C)

Group IV (Received RV for two months, after 4NQO for 20 weeks): Histopathologic results demonstrated moderate dysplasia in the lower two thirds as, basal cell hyperplasia, cellular and nuclear pleomorphism, prominent nucleoli and hyperchromatic nuclei. The underlying connective tissue showed inflammatory cell infiltrate (Figure 5A). Immunohistochemical results revealed a significantly lower mean of mutant p53 positive cells compared to group II, but higher than the 5-FUtreated group (Group III), The mutant p53 positive cells were confined to the basal and suprabasal cells (Figure 5B)(Table 1).

Finally, Group V (received both 5-FU and RV for two months following 4NQO for 20 weeks) showed nearly similar features of (group III) as, slight hyperplasia and thicker keratin layer with mild dysplasia (Figure 6A). Immunohistochemistry revealed a comparable mean of mutant $\mathrm{p} 53$ positive cells to the 5-FU treated group, and were confined to the basal cells only.(Figure 6B,C). (Table 1) 


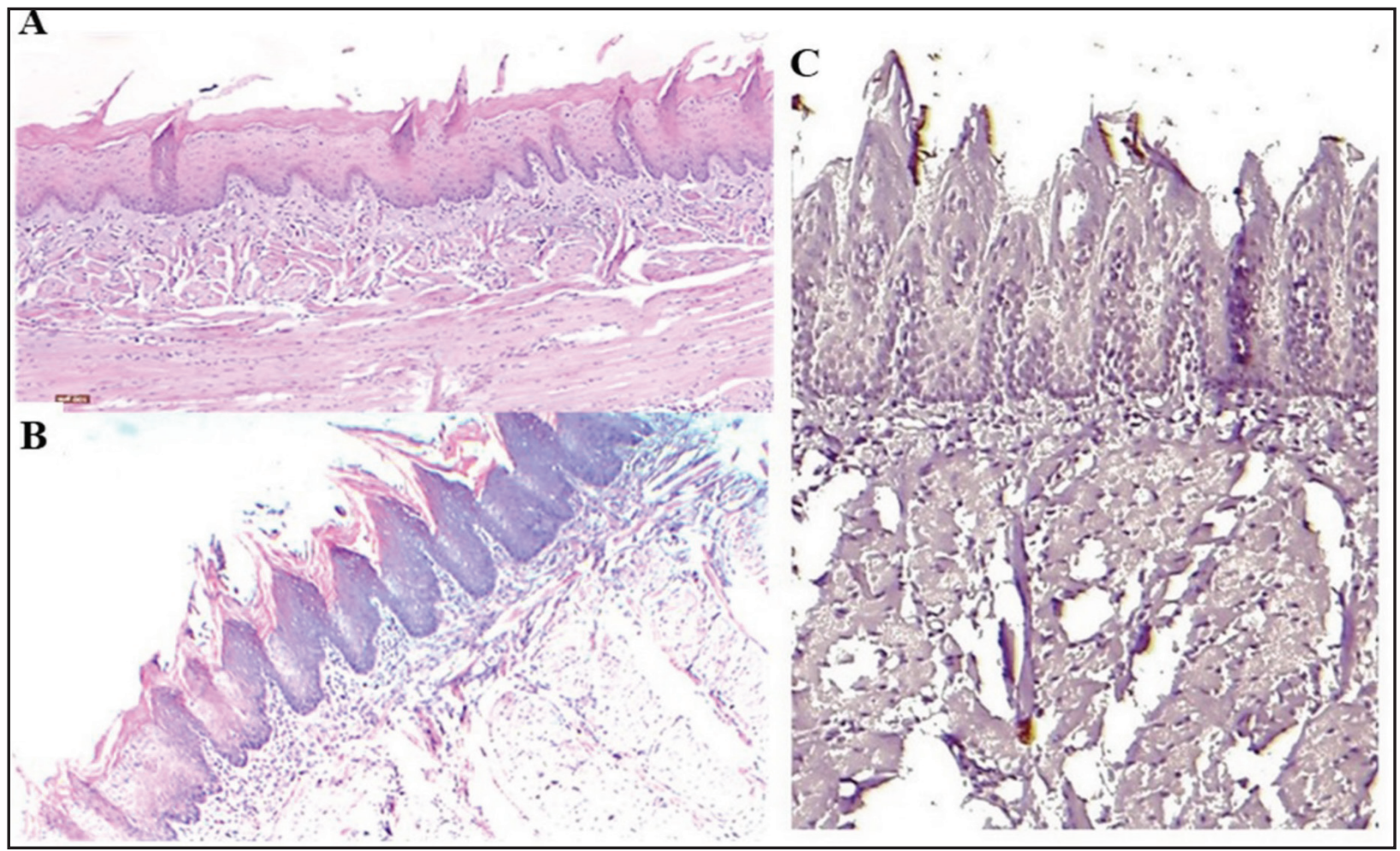

Fig. (1) A photomicrograph of tongue sections from group I (negative control), A, normal rat tongue epithelium, formed of keratinized stratified squamous epithelium, the lamina propria contains dense collagenous fibrous tissue overlying striated muscle fibers (H\&Ex20), B. shows the conical shape keratinized filiform papillae. (H\&E X10).C. mutant p53 IHC-stained tongue section of group I (negative control). Negative p53 immunoreactivity in normal tongue epithelium (IHCX20).

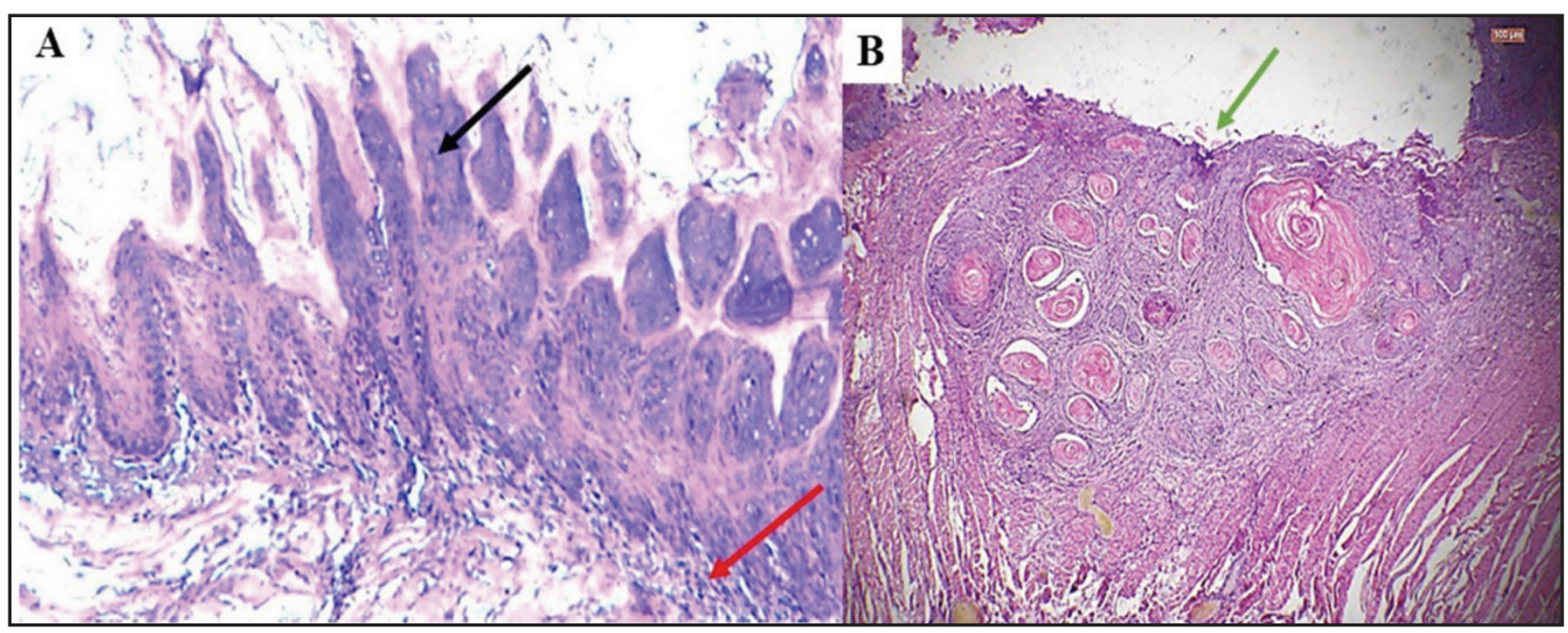

Fig. (2) Photomicrograph of tongue sections from group II (4NQO). A. hyperplasia and hyperkeratinization of the filli-form papillae, with severe dyplasia (black arrow). The underlying connective tissue shows inflammatory cell infiltrate (red arrow). (H\&E $\mathrm{x} 20$ ), B. malignant ulcer of a well/moderately differentiated squamous cell carcinoma ( green arrow), with invading islands and keratin pearls deeper to the muscle layer, with inflammatory infiltrate in the underlying connective tissue (H\&E X10) 


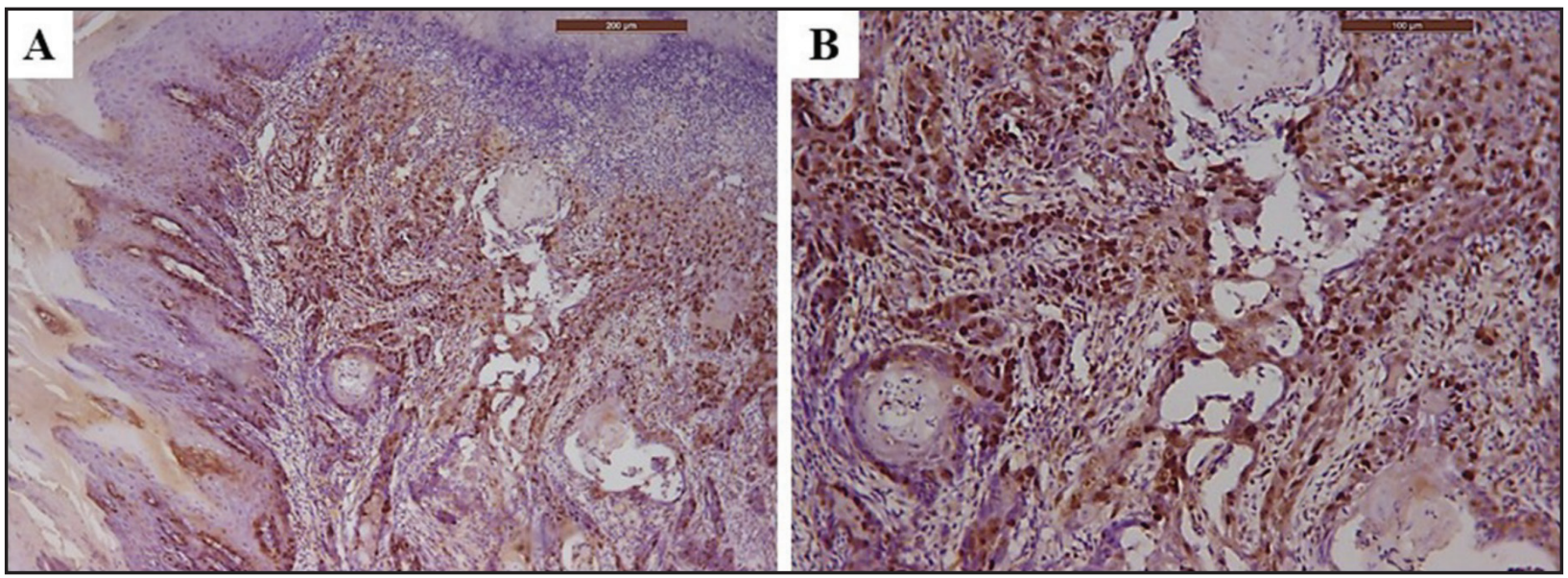

Fig. (3) Photomicrographs of mutant p53 IHC-stained tongue sections from group II showing, A. increase number of mutant p53 positive cells in the surface epithelium and invading epithelial nests (IHC X10), B. Higher power of group II (IHCx20).

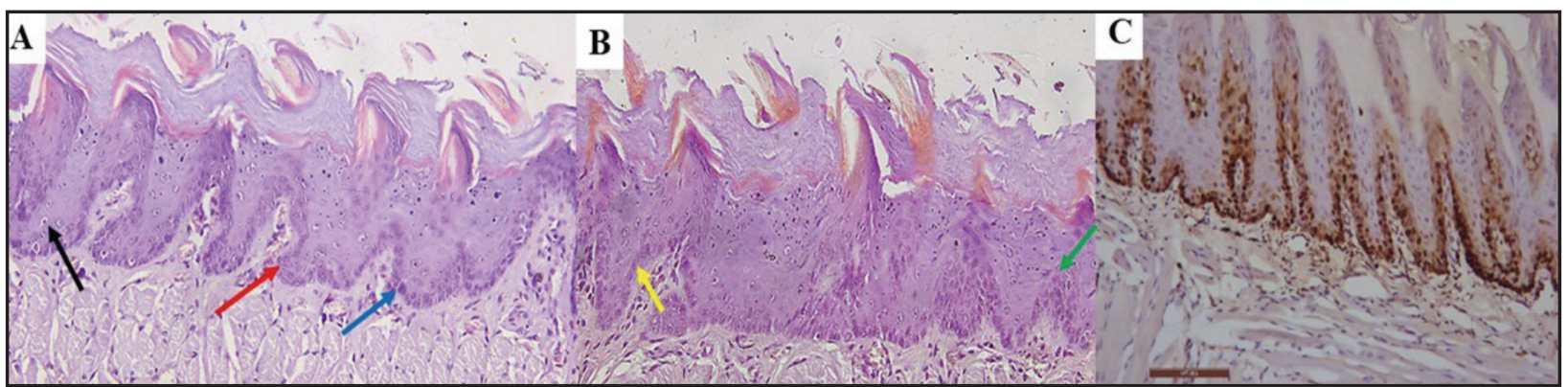

Fig. (4) (A and B), Photomicrographs of tongue sections from group III receiving (5-FU). Showing epithelial hyperplasia and hyperkeratinization with mild dysplasia as, loss of basal cell polarity (yellow arrow), drop shaped rete ridges (red arrow), hyperplasia of basal cell layer (green arrow), nuclear hyperchromatism (blue arrow), prominent nucleoli (black arrow), nuclear and cellular pleomorphism (yellow arrow) (H\&E x20). C, a photomicrograph of mutant p53 IHC-stained tongue section from group III, representing mutant p53 positive cells confined to the basal cell layer (IHCX20).

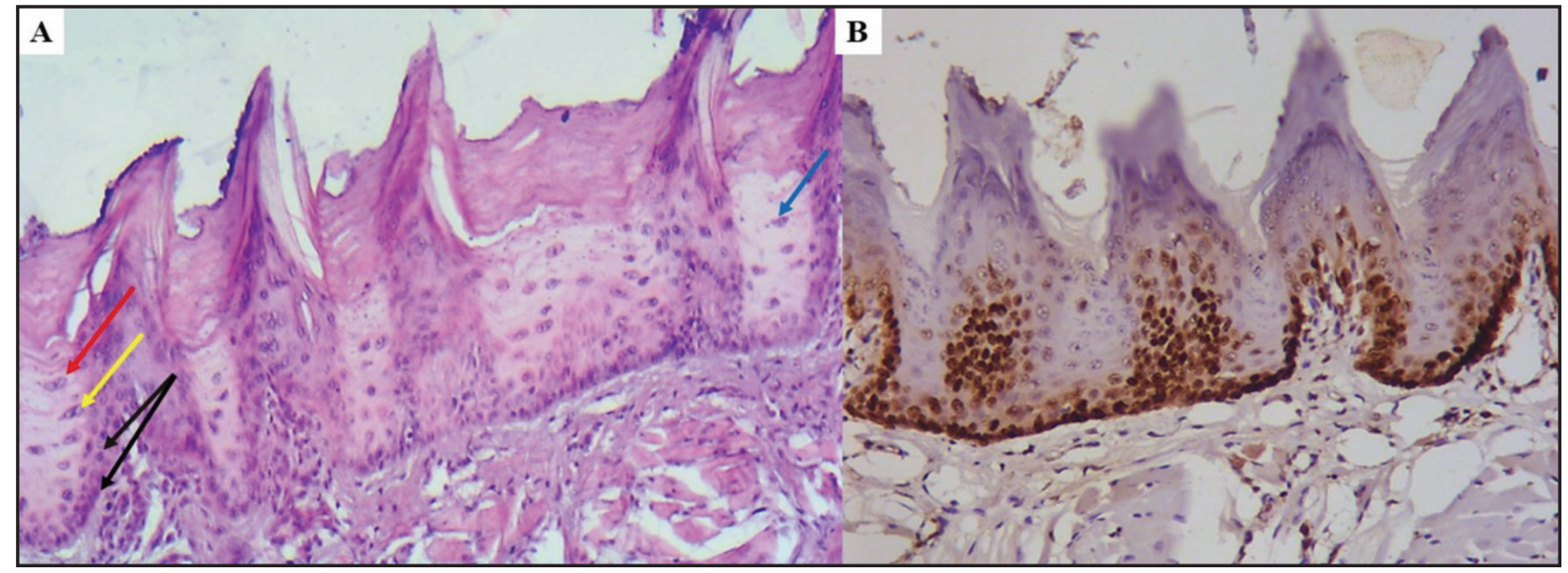

Fig. (5) A, a photomicrograph of tongue section from group IV (receiving RV) showing, hyperkeratinization and hyperplasia, with moderate dysplasia to the middle $1 / 3$ of epithelium, as basal cell hyperplasia (black arrows), prominent nucleoli (yellow arrow), cellular/nuclear pleomorphism (red arrow) and nuclear hyperchromatism (blue arrow). (H\&E X20). B. a photomicrograph of 553 IHC tongue section from group IV, representing mutant p53 positive cells, where the nuclear reaction was confined to the basal and supra basal cells. (IHCX20) 


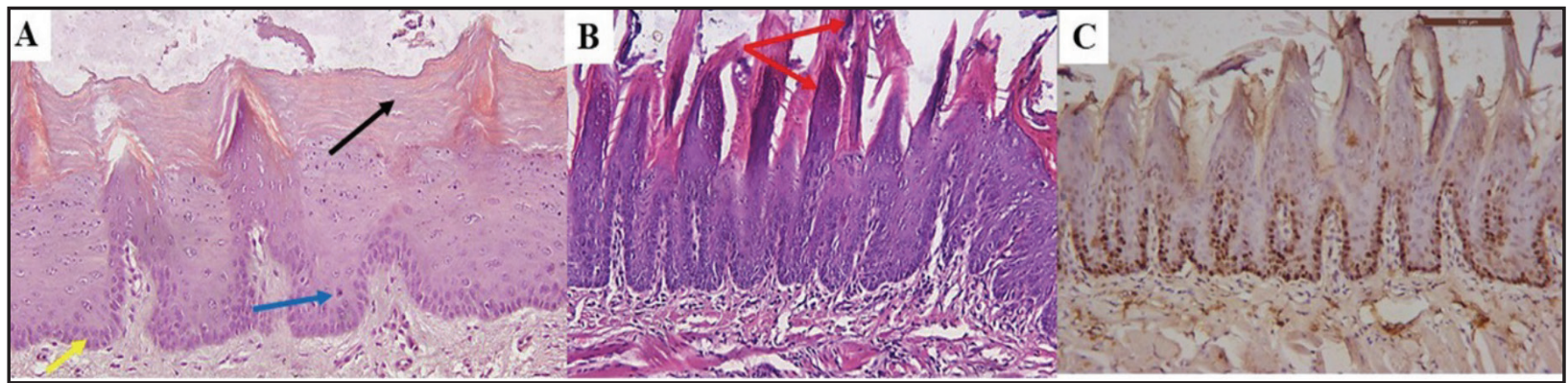

Fig. (6) Photomicrographs of tongue sections from group V (receiving both (RV) and (5FU). A, Showing epithelial hyperplasia and hyperkeratinization (black arrow) with mild dysplasia confined to the basal one third, as basal cell hyperplasia (yellow arrow) and increased nuclear/ cytoplasmic ratio (blue arrow). B, Showing hyperplasia and hyperkeratinization of the filiform papillae (red arrows) (H\&EX20).C,Showing mutant p53 IHC-stained tongue section from group V, representing mutant p53 positive cells, where the nuclear reaction is confined to the basal cells (arrow)(IHCX20).

\section{Statistical analysis of p53 immune expression}

Numerical data were explored for normality by checking the distribution of data and using tests of normality (Kolmogorov-Smirnov and Shapiro-Wilk tests). Number of positive cells data showed nonnormal (non-parametric) distribution while weight, ALT, AST and creatinine level data showed normal (parametric) distribution. Data were presented as mean, \pm standard deviation (SD), median and range values. For non-parametric data, Kruskal-Wallis test was used to compare between groups. Dunn's test was used for pair-wise comparisons when KruskalWallis test is significant. For parametric data, oneway ANOVA test was used to compare between groups. Tukey's (post-hoc test) was used for pairwise comparisons when ANOVA test is significant. Spearman's correlation coefficient was used to determine significant correlation between different variables. Qualitative data were presented as frequencies and percentages. Fisher's Exact test was used to compare between groups. The significance level was set at $p \leq 0.001$. Statistical analysis was performed with IBM SPSS Statistics for Windows, Version 23.0. Armonk, NY: IBM Corp.

\subsection{Number of positive cells: (Table 1)}

There was a statistically significant difference between the groups $(P$-value $\leq 0.05$, Effect size $=$ $0.843)$. Pair-wise comparisons between the groups revealed that positive control group II, showed the statistically significant highest mean number of cells. Resveratrol (group IV) showed statistically significant lower mean value. There was no statistically significant difference between (5-FU) (group III), and (resveratrol and fluorouracil) (group V), where both showed statistically significant lower mean number of positive cells. Negative control group showed the statistically significant lowest mean number of positive cells (Figure 7A).

Table 1: Descriptive statistics and results of Kruskal-Wallis test for comparison between number of p53 positive cells in all groups.

\begin{tabular}{|c|c|c|c|c|}
\hline Group & Mean & SD & $P$-value & $\begin{array}{c}\text { Effect size } \\
\text { (Partial eta squared) }\end{array}$ \\
\hline Group I & $1.8^{\mathrm{D}}$ & 1.3 & & \\
\cline { 1 - 2 } Group II & $126^{\mathrm{A}}$ & 8.5 & \multirow{3}{*}{$\leq 0.001^{*}$} & \multirow{2}{*}{0.843} \\
\cline { 1 - 2 } Group III & $39.4^{\mathrm{C}}$ & 3.8 & \\
\hline Group IV & $56.5^{\text {В }}$ & 2.5 & & \\
\hline Group V & $39.5^{\mathrm{C}}$ & 3 & & \\
\hline
\end{tabular}

Different superscripts are statistically significant,

* Significant at $P \leq 0.05$ 


\section{Correlation between number of $\mathrm{p} 53$ positive cells and animal's weight (Figure 7A)}

There was a statistically significant inverse (negative) correlation between weight and number of p53 positive cells ( $\mathrm{Q}=-0.596, P$-value $\leq 0.05$ ). An increase in weight is associated with a decrease in number of $\mathrm{p} 53$ positive cells.
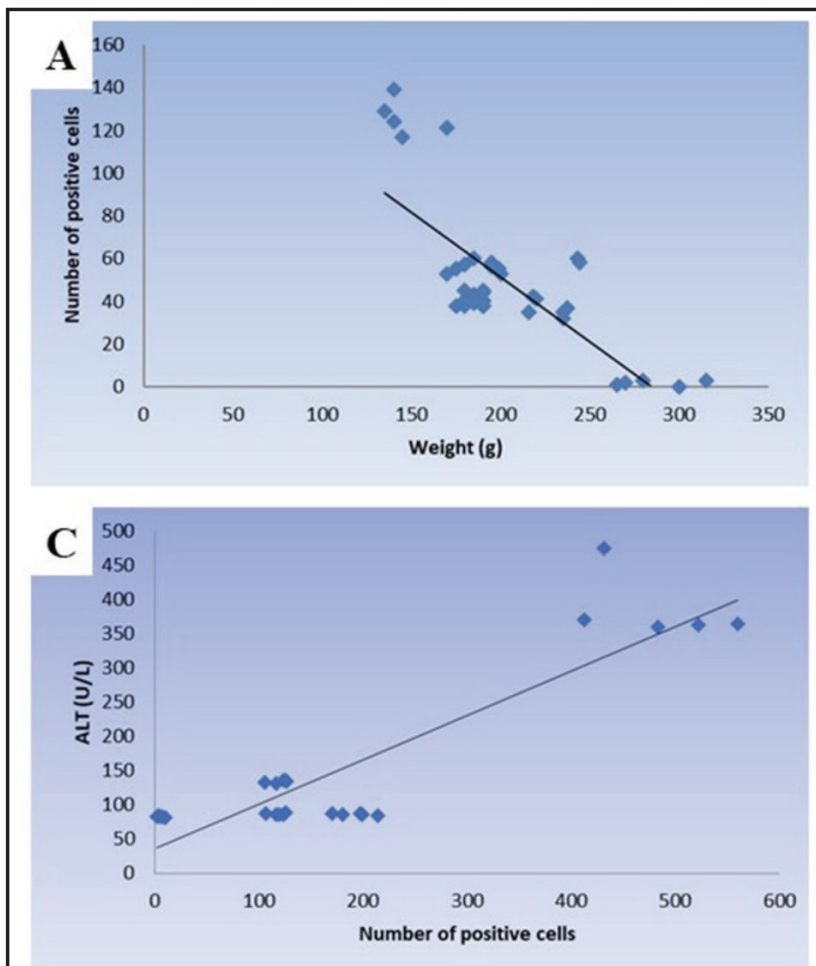

\section{Correlation between number of $\mathrm{p53}$ positive cells and toxicity tests: (Figures 7 B-D)}

There was a statistically significant direct (positive) correlation between kidney and liver enzyme analysis (serum creatinine, ALT and AST) respectively, with the number of $\mathrm{p} 53$ positive cells ( $\varrho=0.596$, P-value $\leq 0.05)$. An increase in enzyme levels is associated with an increase in number of p53 positive cells.
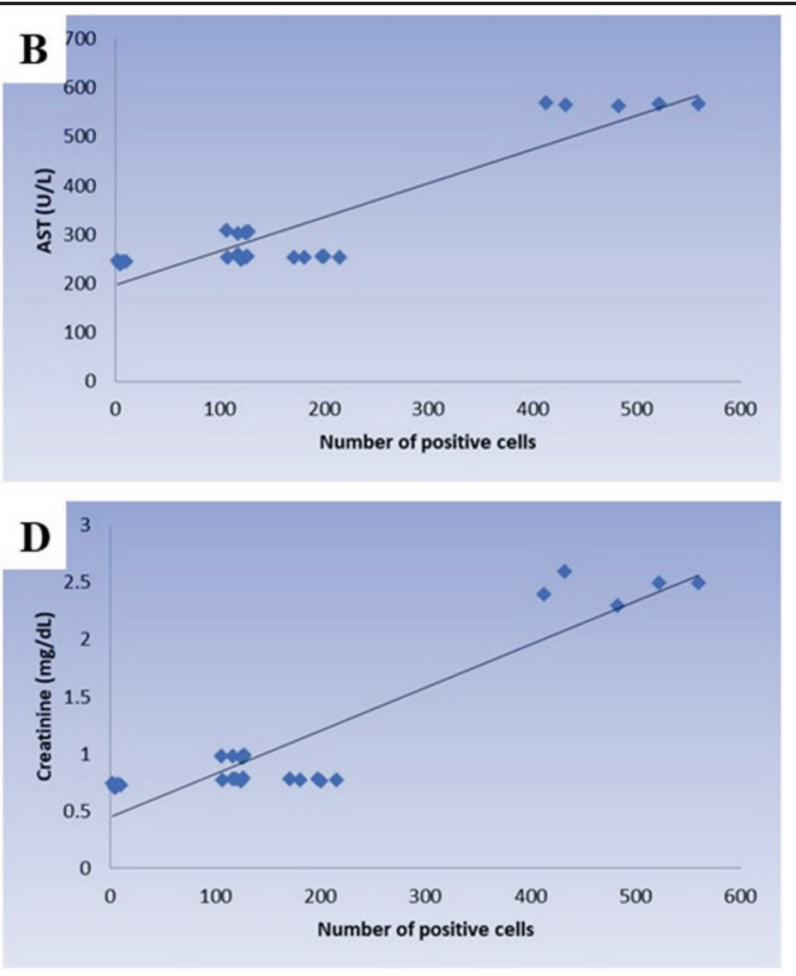

Fig. (7) Scatter diagram representing, A, Inverse correlation between weight and number of p53 positive cells. Statistically significance at $\mathrm{p} \leq 0.05 \mathrm{~B}$, Correlation between serum creatinine level and number of positive cells. Statistically significance at $\mathrm{p} \leq 0.05 \mathbf{C}$, Correlation between AST levels and number of positive cells. Statistically significance at $\mathrm{p} \leq 0.05 \mathbf{D}$. Correlation between ALT and number of positive cells. Statistically significance at $\mathrm{p} \leq 0.05$

\section{DISCUSSION}

Through this study the anticancer effects of resveratrol alone or combined with 5-FU were assessed on induced tongue carcinoma in albino rats.

Serum blood analysis of ALT, AST and creatinine to detect liver and kidney toxicity before euthanization of all animals were analyzed. Significantly high levels of ALT, AST and serum creatinine were detected in animals of group II as compared to group I. The previous observation confirmed the toxic effect of 4NQO.

Serum blood analysis of group III (5-FU) resulted in a significant toxicity for liver and kidney but still not comparable to group II, which was highly significant. 
Animals of group IV and V revealed the least toxicity to the liver and kidney, proving that resveratrol has the least significant toxicity, thereby it diminished the cytotoxic effect of 5-FU.

These observations are in accordance with Madankumar et $a l . .^{(22)}$, in a rat cancer induction study by 4 NQO (in drinking water) in a dose of 50 ppm. They documented that all rats were generally debilitated with reduced hemoglobin levels and aplastic anemia, in addition to high mortality rate due to the toxic effect on liver and kidney.

Another study by Carneiro-Filho et al. ${ }^{(23)} 5$-FU used for treatment of oral, colon, esophageal, stomach and pancreatic cancers, still carries side effects as liver and kidney toxicity, hair fall as well as loss of body weight.

In a study by Crowell et al. ${ }^{(23)}$, to detect renal toxicity of resveratrol at different doses on rats , by measuring kidney enzymes. They concluded that resveratrol has no side effects on kidneys in all doses used, except extremely high doses reaching $300 \mathrm{mg} /$ $\mathrm{kg}$. This dose caused elevation of kidney enzymes, in comparison to the dose used in the current study.

Five animals were selected as negative control (Group I), were clinically healthy, without any signs of debilitation, the tongue and perioral tissues were healthy without any inflammations or ulcerations till end of the experiment.

As for groups (II, III, IV and V); the tumor induction protocol of Vered et al. ${ }^{(25)}$ and Ribeiro et al. ${ }^{(26)}$ was used.

In brief, 4NQO in drinking water, in a dose of $50 \mathrm{ppm}$ for twenty weeks, resulted in multiple tongue carcinomas, clinically in the form of malignant ulcers on the dorsal surface, with loss of normal tongue architecture, as atrophy of tongue papillae with general severe edema and erythema. However, not all animals developed identical appearance of malignancy at the same time, possibly because all rats didn't drink equal amounts of water where the carcinogen is dissolved in.

These observations were in accordance with Madankumar et al. ${ }^{(22)}$, who used the same induction protocol on male Wistar rats. They documented that 4NQO provoked multiple tongue malignancies after twenty weeks.

The general debilitation of rats received 4NQO (Group II), were also similar to reports by Liu et $a l .{ }^{(27)}$ The authors used the same induction protocol on a rat model with a dose of 20 ppm for 32 weeks. Erosions, leukoplakia, papillomas and malignant ulcerations appeared after the ninth, twelfth and twentieth week, respectively. The overall debilitated appearance of rats, have been mentioned, indicating 4NQO toxicity as well as its immune suppressive effect.

As for treatment groups, animals treated with 5-FU (Group III), resulted in reduced clinical manifestations, as partial regain of normal tongue architecture, the ulcerations were markedly decreased in comparison to group II. These results are in agreement with Andreadis et al. ${ }^{(28)}$, who studied the effect of 5-FU and cisplatin in advanced OSCC, in human. They stated that the 5-FU treated group showed a marked regression of tumor size, however, many side effects have been reported as general weight loss, nausea, vomiting, myelotoxicity, liver and kidney toxicity.

On the other hand, animals treated with resveratrol (Group IV), revealed less liver and kidney toxicity than animals treated with 5-FU (group III). The animals partially regained their weight to near animals of group I (untreated group). Clinically, the tongues and perioral tissues showed remarkable regression of ulcerations, edema, erythema and started to regain their normal architecture. 
An explanation of the effective therapeutic effect of resveratrol could be that reported by Carbo et al ${ }^{(29)}$. They observed, in a resveratrol study, that rats inoculated with a rapidly growing tumor (Yoshida AH-130 ascites hepatoma), caused a significant regression (25\%) in tumor size. Interestingly, flow cytometric analysis of the tumor cell population declared the existence of an aneuploidy peak (representing 28\% of total), suggesting that resveratrol causes apoptosis resulting in a decreased tumor cell number. Another in-vivo study by Garvin $e t$ $a l .{ }^{(30)}$ in human breast cancer xenografts treated with resveratrol, showed a decrease in the tumor growth, decreased angiogenesis and increased apoptotic index in resveratrol-treated nude mice compared with controls.

Animals treated with both 5-FU and resveratrol (group V), according to the present results, showed a remarkable weight gain in comparison to groups II and III. Moreover, marked reduction of tongue edema, erythema and ulcerations, despite decreasing the dose of 5-FU and resveratrol to their half.

The present results are online with Abdelatif et al. ${ }^{(31)}$; who studied the combined therapy of resveratrol and 5-FU in treatment of colon cancer in rats. They stated that this combination was effective in regression of colon cancer and less debilitation in comparison to the groups treated with 5-FU alone.

Results of the present work are also in accordance with Soliman et al..$^{(4)}$ study, who examined combined 5-FU and resveratrol, in colorectal cancer of rats. They reported marked decrease of general debilitation than those treated with 5-FU alone. The authors explained the enhancing effect of resveratrol on 5-FU treatment could be due to reducing cytotoxicity of a decreased dose of 5-FU to half.

As clinical observations mostly reflect the histopathological findings, the following results were obtained. Animals of group I (the untreated group), showed normal tongue mucosa without any signs of dysplasia. Regarding their immunohistochemical observations in the present work, negative mutant p53 expression was revealed in that group.

These results are in accordance with Hasty and Christy ${ }^{(32)}$. As p53 is well known for suppressing tumors, it responds to a variety of stresses to either induce apoptosis or cell cycle arrest. Therefore, p53 is not mutated under normal physiological conditions $^{(33)}$.

As for Group II animals, where cancer induction by 4 NQO had been developed at the twentieth week. Immuntohistochemical results revealed the highest mean of mutant p53 positive cells, in accordance with Liu et al. ${ }^{(27)}$.

According to Madankumar et al. ${ }^{(22)}$, 4NQO developed well to moderately differentiated SCC after week twenty, by upregulating the expression of p65 (subunit of NF- $x \mathrm{~B}$ ) in the nucleus, TNF- $\alpha$, IL-1 $\beta$, COX-2 and iNOS.

In another study by Osugi ${ }^{(34)}$, focused on p53 expression in different stages of 4-nitroquinoline 1-oxide- induced carcinoma in the rat tongues. He documented that mutant $\mathrm{p} 53$-positive epithelial cells increased in direct proportion to development of the tumor. Significant increase was observed during the eighth to twelfth week after 4NQO administration and reached the highest significance of tumor size and $\mathrm{p} 53$ positive cells after the twentieth week.

Animals treated with 5-FU (Group III) in the present work, showed mild to moderate dysplastic changes of the tongue epithelium with a siginificantly lower mean of mutant p53 compared to group II. These results are comparable to Soliman et al. ${ }^{(4)}$, they documented that the 5-FU-treated group showed mild dysplastic changes of colon cells than those in the untreated colon cancer induction group, which showed invasive SCC. 
Animals treated with resveratrol (Group IV), showed moderate dysplasia of the tongue epithelium, with a significantly lower mean value of mutant p53 positive cells compared to group II.

A study by Ko et $a l^{(10)}$, showed that resveratrol has regressed various cancer cell lines and from dividing indefinitely. They explained that due to the ability of resveratrol to avoid carcinogenesis through the relief of oxidative stress and cancer-cell proliferation, as well as the activation of regulated cell death mechanisms. Furthermore, Baur and Sinclair ${ }^{(11)}$, had stated that resveratrol binds to various cell-signaling molecules and modulates cell-cycle regulatory genes. It activates transcription factors, inhibits protein kinases and expression of antiapoptotic genes, angiogenic and metastatic gene products as well as, inflammatory biomarkers. It also induces antioxidant enzymes and alters the expression of enzymes such as cytochrome P450s that are involved in drug metabolism ${ }^{(11)}$.

The mutant p53 expression in group IV (resveratrol-treated), was comparable to a study done by Ferraz da Costa et al. They stated that localization of mutant p53 with amyloid aggregates in MDA-MB-231 human breast cancer cells, after treatment by resveratrol $(50$ and $100 \mu \mathrm{m})$ for $24 \mathrm{~h}$, promoted a significant $(\mathrm{p} \leq 0.05)$ reduction in nuclear $\mathrm{p} 53$ aggregate formation ${ }^{(35)}$.

Finally group V, treated with half dose of both resveratrol and 5-FU, showed in histologic sections mild dysplasia, in comparison to other treated groups.

These results are comparable to Soliman et $a l .{ }^{(4)}$ study. They stated that resveratrol has an antiproliferative activity against colorectal cancer invivo and enhanced the therapeutic effect of 5-FU. In another study by Chung et al. They stated that the combinational therapy of resveratrol with $5-\mathrm{FU}$ in colon cancer cell lines resulted in reduction of tumor cell number and cytotoxicity ${ }^{(36)}$.

The expression of mutant p53 in that group revealed a comparable mean number to group III (5FU), this may be an indication of enhancing action of resveratrol to 5-FU due to reducing the dose of 5-FU, as compared to animals treated with 5-FU alone.

These results are comparable to Chan et al. in an in vivo study of combinational therapy of resveratrol with 5-FU in colon cancer. They concluded that resveratrol combinational therapy promotes $5-\mathrm{FU}$ action, it also triggered 5-FU- induced apoptosis, in turn reduces the number of $\mathrm{p} 53$ positive cells ${ }^{(37)}$.

Also, in accordance with Wu et al., who studied the combinational effect of 5-FU and resveratrol on murine liver cancer (H22 murine hepatoma). They concluded that resveratrol could induce the $S$ phase arrest of hepatoma cells, enhance the antitumor effect of 5-FU on murine hepatoma $\mathrm{H} 22$ and markedly antagonize its toxicity ${ }^{(38)}$.

The present results suggest that resveratrol, as a biochemical modulator to enhance the therapeutic effects of 5-FU, may be potentially useful in cancer chemotherapy with reduction of 5-FU dose, as well as minimizing the duration of treatment.

Finally, resveratrol mode of action could be through decreasing angiogenesis, increasing apoptotic index and inhibiting protein kinases ${ }^{(30)}$. Adding to the mechanism of 5-FU which is principally a thymidylate synthase (TS)- inhibitor leading to interruption of DNA replication ${ }^{(39)}$. Supporting the use of both drugs in combination with reduction of 5-FU dose to half to minimize toxicity and duration of oral cancer therapy. 


\section{CONCLUSION}

1. Resveratrol has a beneficial therapeutic effect against the strong cytotoxic and carcinogenic action of $4 \mathrm{NQO}$ in that model.

2. Resveratrol is a safe natural agent for enhancing chemotherapy with 5-FU in that model.

3. Resveratrol helped in lowering 5-FU dose to half with the same therapeutic effect on SCC treatment with lower toxicity in that model.

4. Combination therapy with 5-fluorouracil and resveratrol had regressed the OSCC to mild dysplasia.

\section{ACKNOWLEDGEMENTS}

There was no conflict of interest in the study.

\section{REFERENCES}

1. Marur S, Forastiere AA. Head and neck squamous cell carcinoma: update on epidemiology, diagnosis, and treatment. Mayo Clin Proc 2016;91(3):386-396.

2. Tecza K, Pamula-Pilat J, Lanuszewska J, Butkiewicz D, Grzybowska E. Pharmacogenetics of toxicity of 5-fluorouracil,chemotherapy in breast cancer patients. Oncotarget 2018;9(10):9114-9136.

3. Hwang JT, Kwak DW, Lin SK, Kim HM, Kim YM, Park OJ. Resveratrol induces apoptosis in chemoresistant cancer cells via modulation of AMPK signaling pathway. Ann NY Acad Sci 2007;1095:441-448.

4. Soliman B, Farrag AR, Khaled H. Combinational effect of 5-flourouracil and resveratrol against N-nitroso-Nmethyl urea induced colorectal cancer. Egypt J Hosp Med 2018;70:994-1006.

5. Grem JL. 5-Fluorouracil: Forty-plus and still ticking. A review of its preclinical and clinical development. Invest New Drugs 2000;18(4):299-313.

6. Leichman CG, Fleming TR, Muggia FM, Tangen CM, Ardalan B, Doroshow JH. Phase II study of fluorouracil and its modulation in advanced colorectal cancer: A southwest oncology group study J Clin Oncol. 1995;13(6):13031311.
7. Ong CK, Bodeker C, Grundy C, Burford G, Shein K. WHO Global Atlas of traditional, complementary and alternative medicine 2005; 2(1):120-130.

8. Shu XH, Wang LL, Li H, Song X, Shi S, Gu JY, Wu ML, Chen XY, Kong QY, Liu J. Diffusion efficiency and bioavailability of resveratrol administered to rat brain by different routes:Therapeutic implications. Neurotherapeutics. 2015;12(2):491-501.

9. Bhardwaj A, Sethi G, Vadhan-Raj S, Bueso-Ramos C, Takada Y, Gaur U. Resveratrol inhibits proliferation, induces apoptosis, and overcomes chemoresistance through down-regulation of STAT3 and nuclear factor-kappaBregulated antiapoptotic and cell survival gene products in human multiple myeloma cells Blood 2007;109(6): 2293-2302.

10. Ko JH, Sethi G, Um JY, Shanmugam MK, Arfuso F, Kumar AP. The Role of Resveratrol in Cancer Therapy Int J Mol Sci 2017;18(12):200-221.

11. Baur JA, Sinclair DA. Therapeutic potential of resveratrol: the in vivo evidence. Nat Rev Drug Discov 2006 Jun; 5(6):493-506

12. Gomez-Lazaro M, Fernandez-Gomez FJ, Jordan J. p53: Twenty five years understanding the mechanism of genome protection. J Physiol Biochem 2004 Dec;60(4):287-307.

13. Ogden GR, Chisholm DM, Morris AM, Stevenson JH. Overexpression of p53 in normal oral mucosa of oral cancer patients does not necessarily predict further malignant disease. J Pathol 1997;182(2):180-184.

14. Abbas NF, Labib S, Abbas EA, Abdelmonem M. Immunohistochemical study of p53 and angiogenesis in benign and preneoplastic oral lesions and oral squamous cell carcinoma. Oral Surg Oral Med Oral Pathol Oral Radiol Endod 2007;103(3):385-390.

15. Hawkins BL, Heniford BW, Ackermann DM, Leonberger M, Martinez SA, Hendler FJ. 4NQO carcinogenesis: A mouse model of oral cavity squamous cell carcinoma. Head Neck 1994;16(5):424-432.

16. Kitakawa D, Cabral L, Marques M, Salvadori D, Ribeiro DA. Medium-term tongue carcinogenesis assays: A comparative study between 4-nitroquinoline 1-oxide (4NQO)induced rat and dimethylbenzanthracene (DMBA)-induced hamster carcinogenesis J Exp Anim Sci. 2006;43(2): 219-227. 
17. Rivera C. 4 NQO Carcinogenesis: A model of oral squamous cell carcinoma. Int J Morphol 2012;30(1):309-314.

18. Suzuki R, Kohno H, Suzui M, Yoshimi N, Tsuda H, Wakabayashi K. An animal model for the rapid induction of tongue neoplasms in human c-Ha-ras proto-oncogene transgenic rats by 4-nitroquinoline 1-oxide: its potential use for preclinical chemoprevention studies. Carcinogenesis 2006;27(3):619-630.

19. Boffetta P, Hecht S, Gray N, Gupta P, Straif K. Smokeless tobacco and cancer. Lancet Oncol 2008 Jul;9(7):667-75.

20. El-Naggar AK, Chan JKC, Rubin Grandis J, Takata T, Slootweg PJ, International agency for research on cancer. WHO classification of head and neck tumours. IARC Press 2017;4(9):1-5.

21. Neville B. W. Oral and Maxillofacial Pathology. $4^{\text {th }}$ ed. St. Louis, Mo: Saunders, Elsevier; 2009:605-610.

22. Madankumar A, Tamilarasi S, Premkumar T, Gopikrishnan M, Nagabhishek N, Devaki T. Geraniol attenuates 4NQOinduced tongue carcinogenesis through downregulating the activation of NF-kappaB in rats Mol Cell Biochem 2017;434(2):7-15.

23. Carneiro-Filho BA, Oria RB, Wood Rea K, Brito GAC, Fujii J, Obrig T. Alanyl-glutamine hastens morphologic recovery from 5-fluorouracil-induced mucositis in mice. Nutrition 2004;20(10):934-941.

24. Crowell JA, Korytko PJ, Morrissey RL, Booth TD, Levine BS. Resveratrol-associated renal toxicity. Toxicol Sci 2004;82(2):614-619.

25. Vered M, Daniel N, Hirshberg A, Dayan D. Histomorphologic and morphometric changes in minor salivary glands of the rat tongue during 4-nitroquinoline-1-oxide-induced carcinogenesis. Oral Oncol 2003; 39(5):491-496.

26. Ribeiro DA, Salvadori DMF. Gingival changes in wistar rats after oral treatment with 4-nitroquinoline 1-oxide. Eur J Dent 2007;1(3):152-157.

27. Liu X, He R, Chen W. A rat model of tongue mucosa squamous cell carcinoma induced by oral administration of 4NQO in drinking water. Zhonghua Kou Qiang Yi Xue Za Zhi 1999;34(6):354-356.

28. Andreadis C, Vahtsevanos K, Sidiras T, Thomaidis I, Antoniadis K, Mouratidou D. 5-Fluorouracil and cispla- tin in the treatment of advanced oral cancer. Oral Oncol 2003;39(4):380-385.

29. Carbo N, Costelli P, Baccino FM, Lopez-Soriano FJ, Argiles JM. Resveratrol, a natural product present in wine, decreases tumour growth in a rat tumour model. Biochem Biophys Res Commun 1999;254(3):739-743.

30. Garvin S, Ollinger K, Dabrosin C. Resveratrol induces apoptosis and inhibits angiogenesis in human breast cancer xenografts in vivo. Cancer Lett 2006;231(1):113-122.

31. Abdelatif Y, El-bana M, Hussein J, El-Khayat Z, Farrag AR. Effects of resveratrol in combination with 5-fluorouracil on N-methylnitrosourea-induced colon cancer in rats. Comp Clin Path 2019;4(1):100-110.

32. Hasty P, Christy BA. P53 as an intervention target for cancer and aging. Pathobiol Aging Age-related Dis 2013; 3(1):22702-22709.

33. Beckerman R, Prives C. Transcriptional regulation by P53. Cold Spring Harb Perspect Biol 2010;2(1):a000935a00939.

34. Osugi Y. p53 Expression in various stages of 4-nitroquinoline 1-oxide induced carcinoma in the rat tongue. J Osaka Dent Univ 1996;30(2):29-35.

35. Ferraz da Costa DC, Campos NPC, Santos RA, Guedesda-Silva FH, Martins-Dinis MMDC, Zanphorlin L. Resveratrol prevents p53 aggregation in vitro and in breast cancer cells. Oncotarget 2018;9(49):29112-29122.

36. Chung SS, Dutta P, Austin D, Wang P, Awad A, Vadgama JV. Combination of resveratrol and 5-flurouracil enhanced anti-telomerase activity and apoptosis by inhibiting STAT3 and Akt signaling pathways in human colorectal cancer cells. Oncotarget 2018; 9(68):32943-32957.

37. Chan JY, Phoo MS, Clement M-V, Pervaiz S, Lee SC. Resveratrol displays converse dose-related effects on 5-fluorouracil-evoked colon cancer cell apoptosis: the roles of caspase-6 and p53. Cancer Biol Ther 2008;7(8):1305-1312.

38. Wu SL, Sun ZJ, Yu L, Meng KW, Qin XL, Pan CEEffect of resveratrol and in combination with 5-FU on murine liver cancer. World J Gastroenterol 2004;10(20):3048-3052.

39. Blanke CD, Kasimis B, Schein P, Capizzi R, Kurman M. Phase II study of trimetrexate, fluorouracil, and leucovorin for advanced colorectal cancer. J Clin Oncol 1997;15(3):915-920. 